\title{
Perceptual salience of form versus material as a function of variations in spacing and number of elements
}

\author{
RAYMOND M. KLEIN and JOHN BARRESI \\ Dalhousie University, Halifax, Nova Scotia, Canada
}

\begin{abstract}
When do the elements composing a pattern shift in their functional role from integral parts of its overall form to the material out of which its global form is constructed? In a parametric extension of Goldmeier's (1936/1972) initial work on this problem, subjects judged the similarity between, and serially categorized, pairs of lines that were constructed from element dots such that one line was twice the length of the other, and either maintained the same number of elements or the same spacing between elements. For large stimuli composed of few elements, number of elements rather than spacing determined similarity and categorization time. However, as the number of elements was increased, the two tasks dissociated. Similarity judgments became dependent on spacing between elements rather than on number, whereas categorization time became independent of this factor. This suggests that when they are numerous and closely spaced, the elements shift from integral parts of the figure to independently coded material.
\end{abstract}

One of the most enduring dichotomies in the psychology of perception is epitomized in the debate between the Gestalt and Structuralist schools. Woodworth's summary of the debate is still accurate today, as investigators continue to ask "whether the elements of a figure get together and build up the whole, or whether the whole figure emerges and organizes the available elements" (Woodworth \& Schlosberg, 1954, p. 403). In current jargon, scholars ask whether processing is "bottom-up" or "topdown," or whether "global" analysis precedes or follows "local" analysis.

Historically, phenomenology was the primary tool for answering such questions about the nature of perception. Within this tradition, Goldmeier's (1936/1972) classic studies of perceived similarity, provide a wealth of interesting empirical data and insightful conceptual analysis. In exploring the general proposition that similarity is dependent upon the identity of relations, rather than the identity of elements, Goldmeier found several exceptions, which seemed to depend upon a phenomenal separation of form and material. He proposed that:

(Rule 2) the phenomenal separation of material and form arises in the following manner: Characteristics of the design which (a) because of relative smallness are not phenomenally related to the overall measurements of the figure as a whole, and which (b), in the case of discon-

This paper was presented at the annual meeting of the Psychonomic Society, November 1983. The research reported here was supported by Natural Sciences and Engineering Research Council of Canada Grants A0667 to John Barresi and A9948 to Raymond Klein. Requests for reprints should be sent to $R$. Klein, Department of Psychology, Dalhousie University, Halifax, Nova Scotia, Canada B3H 4J1. tinuity, are not individualized because of their large number, are relegated to the role of material ... (Rule 1) The form is best preserved by proportional enlargement; material properties are best preserved by keeping the measurements of the material elements constant. (p. 46; 43)

Rule 1 applies only to stimuli in which form and material are phenomenally separate (Rule 2); when they are not separate, proportional enlargement should produce the highest similarity.

In a typical, and for this paper, basic experiment, Goldmeier's observers selected the comparison stimuli more similar to each standard in Figure $1 \mathrm{a}$ and $1 \mathrm{~b}$ (corresponding to Goldmeier's Figures 24 and 25).

As expected from rule $2 b$, if the number of elements is small the enlargement of the figure must be proportional (25b), while if the elements are numerous an impression of material is created which is better rendered in an unproportionally enlarged comparison stimulus $(24 \mathrm{c})$ than in a proportionally enlarged one. (p. 49)

Although Goldmeier concludes that " it is not possible to designate a definite least number of elements necessary for the impression of material," he does conclude that the crossover point, when material emerges, is between 6 and 9 elements.

In recent years mental chronometry has gained favor as a tool (e.g., Garner, 1974; Posner, 1978) and has been profitably used, often in conjunction with phenomenology (e.g., Palmer, 1977), to explore perception. Our principal purpose in this study was to explore the generality of Goldmeier's rules regarding "form" and "material" by parametrically extending his observations from Figures 24 and 25 , and by supplementing the phenomenological 


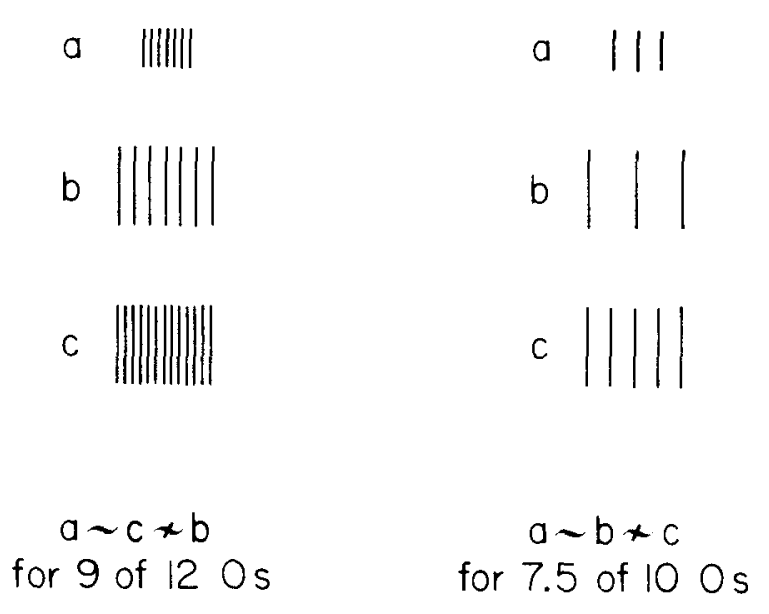

Figure 1. Stimuli and results from Goldmeier's Experiments 24 and 25 (see text for explanation).

data from similarity judgments with performance data (RT and accuracy) from a categorization task.

Goldmeier's work on the form/material distinction has also been extended by Kimchi and Palmer (1982). Although our experiment was conducted independently of theirs, it is worth noting the similarities and differences between our two approaches. Theoretically, there is much in common, as will be pointed out in the discussion section. But empirically there are large and important differences. These occur in both stimulus conditions and response measures. Kimchi and Palmer focused their attention on varying the size, form, and number of the elements out of which the global figures were constructed. Hence, a large square might be constructed out of small or large triangles. In the present study, all of our elements were dots of uniform size and all of our figures were straight lines composed of these dots. Although in both studies the effects of number and spacing of elements were investigated, this was not the major thrust of the Kimchi and Palmer study; nor were their results with this manipulation entirely consistent. The present study investigated this single issue thoroughly within a wide range of psychophysical conditions.

The two studies also differed in response measures. In four out of five of their experiments, Kimchi and Palmer used the original Goldmeier forced-choice similarity task. In the fifth experiment, they asked subjects to verbally describe their figures. In the present study, we used continuous similarity ratings and a parallel performance measure (speeded classification). Although we see little difference between the use of the forced-choice and ratings procedures (apart from the increased confidence and generalizability that derives from replication), the use of performance measures confers specific advantages. Kimchi and Palmer address most of their theoretical remarks to recent developments in the global/local debate. Yet, nearly all the studies they cite on this issue used performance measures rather than verbal reports of similarity or the like. It is not clear a priori that there will be comparable results on both types of measure. Indeed, in earlier work on perceptual structure, Palmer overcame his concern that verbal measures "are subject to the objection that such conscious judgments may have little in common with unconscious perceptual processing'" (Palmer, 1977, p. 456) by exploring the convergence between phenomenology and a variety of performance measures. The present study used perceived similarity together with performance on a speeded classification task, at least partly because we share this concern. As we shall see, differences do occur, and these seem to have theoretical significance.

\section{METHOD}

\section{Stimuli}

The stimuli, shown in Figure 2, were lines composed of from 2-17 dots with a constant distance between dots in a given line. Each triplet in this half matrix represents two stimulus conditions experienced by all subjects. The subjects judged the similarity between the standard stimulus (middle member of triplet) and a comparison stimulus, and then performed a speeded classification task with the same pair. Note that the stimulus pairs presented to the subjects always differed by a factor of 2 in total extent, and were either (1) identical in number and different in spacing (above), or (2) identical in spacing and different in number (below). Interdot spacing varied from approximately $.05^{\circ}$ for the smallest spacing used to about $.8^{\circ}$ for the largest spacing. Two groups, differing only in absolute size (see Design), were tested.

These stimuli are analogous to Goldmeier's line stimuli (Figures 24 and 25), except that dots have been substituted for lines. When number (or spacing) is the perceptually salient dimension, pairs that do not differ in number (or spacing) will be perceived as more similar than corresponding pairs that do. Goldmeier's experiments suggest that for linear stimuli those with $3 / 5$ elements are dominated by form (the proportional enlargement ${ }^{1}$ with different spacing is chosen as more similar than the enlargement with the same spacing), whereas with $7 / 13$ elements, material has emerged as perceptually salient (and the opposite selection is made).

\section{Subjects}

Eighteen college students participated for course credit.

\section{Tasks}

Similarity. A stimulus pair was presented with the shorter stimulus on the left side of the screen and the longer one on the right together with a horizontal line marked "low" on the left end and "hi" on the right end. By moving a joystick, the subjects could position a marker anywhere on this line. They were instructed that they should determine the perceived similarity between the two stimuli, place the marker at an appropriate position, and then press a microswitch to indicate that their judgment had been made. The subjects were told to pay special attention to the spatial locations of the two stimuli, since this information would be required during the sorting task.

Sorting. Immediately after the subjects made their similarity ratings, the word "ready" was displayed on the screen until a response was made. Then each of the two stimuli was presented 25 times in a serial RT sorting task. In response to each stimulus, the subject had to depress the microswitch corresponding to the side of presentation of that stimulus during the similarity procedure (for all such tests, the left key was used for the smaller stimulus). Immediately after each response, the next stimulus was presented. The identity and position ${ }^{2}$ of the stimuli was varied randomly from trial to trial. At the end of each set of $\mathbf{5 0}$ trials, the subjects received terminal feedback on their RT and accuracy. 


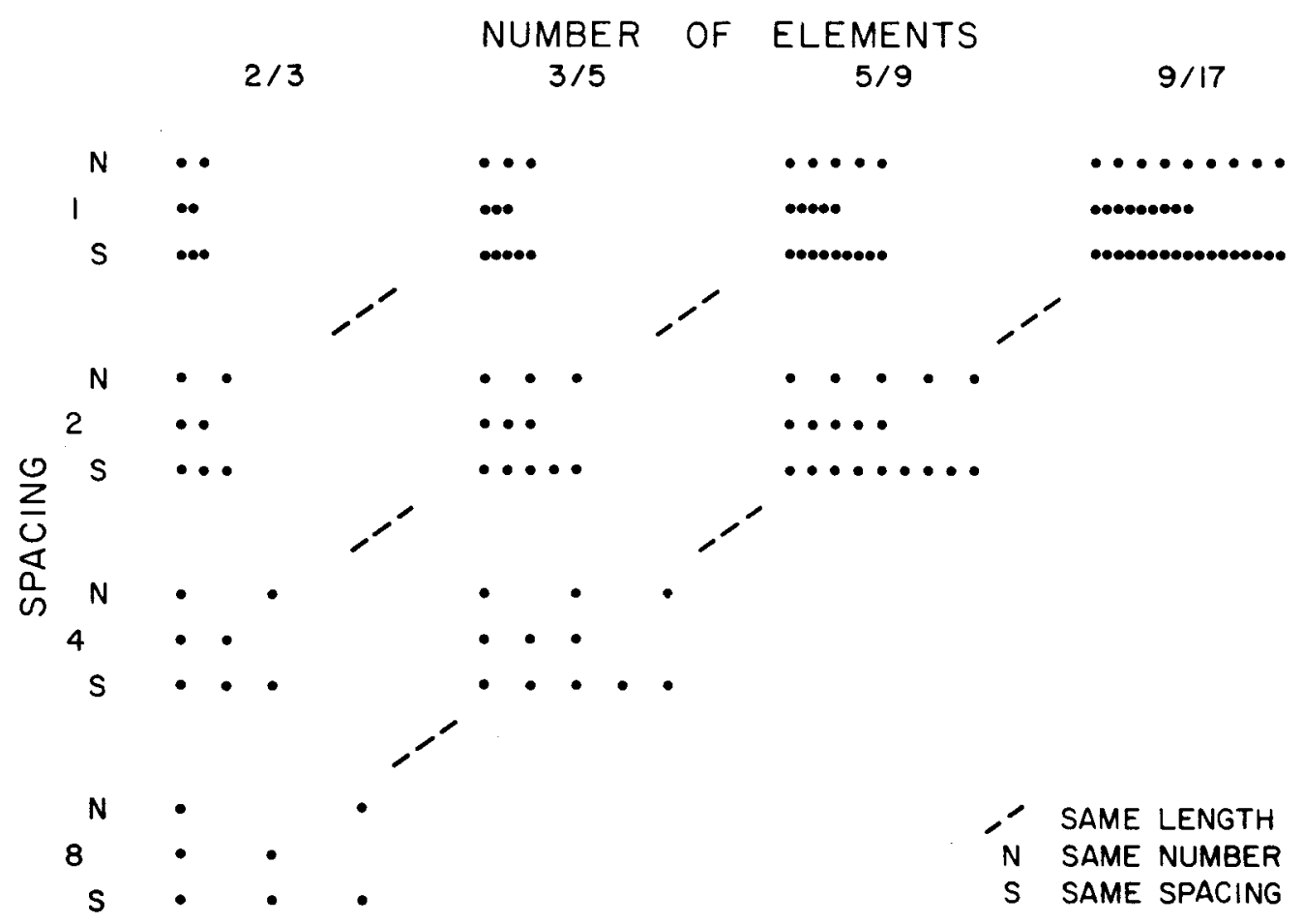

Figure 2. Stimuli used in the present study (see text for explanation).

\section{Procedure}

Each subject rated the similarity between a pair of stimuli and then performed the sorting task. This was done for each of the $\mathbf{2 0}$ pairs shown in Figure 1, and then this was repeated a second time. The subjects were told to try to use a range of similarity values, and to respond as accurately and as rapidly as possible. The order of testing was varied randomly within and between subjects.

\section{Design}

Two groups of subjects ( 10 in small; 8 in large) were treated identically except for the fact that the spacing size was doubled for one group. Thus, base spacing was a between-subjects variable; block (first vs. second exposure), type of comparison stimulus (spacing or number varies), and triplet (the 10 unique combinations of number and spacing, see Figure 2) were within-subjects variables.

\section{BDSULTS}

The results of one subject from the large group were not included in the analysis because his similarity ratings did not discriminate between the stimuli, with most pairs rated "identical."

Overall ANOVAS were conducted on the similarity ratings, latencies, and error rates. In these ANOVAS, base spacing was a between-subjects variable and blocks, type of comparison stimulus, and triplet were within-subject variables. The significant effects are summarized in Table 1 . Although there are highly significant trends as a function of spacing, number, and overall size ${ }^{3}$ of the various stimulus pairs, in none of these analyses was there a main effect of groups, and there were no significant interactions involving groups and type of comparison stimulus or groups and triplet. Although this failure of groups to interact with triplet suggests that the effects of spacing and number might be due to relative (context sensitive), rather than absolute (retinal), size, two points militate against a firm endorsement of this view. First, base spacing was a between-subjects manipulation and, with only 7 and 10 subjects per group, there may not have been sufficient power to detect the pattern of interactions compatible with a role for retinal size. Second, since we did not use a chinrest, viewing distance, and therefore retinal size, was not completely under experimental control.

Our focus in the remainder of the results section will be to examine triplets of the same size (diagonals) to determine the crossover point (in number/spacing) where material emerges and similarity is no longer dominated

Trate 1

Significant Etiects in Doch of the Three Overnd ANOVAs

\begin{tabular}{|c|c|c|c|}
\hline & df & $\mathbf{F}$ & $\mathbf{p}$ \\
\hline \multicolumn{4}{|c|}{ Similarity } \\
\hline $\begin{array}{l}\text { Comparison } \\
\text { Triplet } \\
\text { Comparison } \times \text { Triplet }\end{array}$ & $\begin{array}{l}1,15 \\
9,135 \\
9,135\end{array}$ & $\begin{array}{r}5.39 \\
19.63 \\
9.72\end{array}$ & $\begin{array}{l}.05 \\
.001 \\
.001\end{array}$ \\
\hline \multicolumn{4}{|c|}{ Categorization Time } \\
\hline $\begin{array}{l}\text { Block } \\
\text { Comparison } \\
\text { Triplet } \\
\text { Comparison } \times \text { Triplet }\end{array}$ & $\begin{array}{l}1,15 \\
9,135 \\
9,135 \\
9,135\end{array}$ & $\begin{array}{r}51.94 \\
30.83 \\
31.51 \\
2.80\end{array}$ & $\begin{array}{l}.001 \\
.001 \\
.001 \\
.01\end{array}$ \\
\hline \multicolumn{4}{|c|}{ Categorization Accuracy } \\
\hline $\begin{array}{l}\text { Triplet } \\
\text { Group } \times \text { Block }\end{array}$ & $\begin{array}{l}9,135 \\
1,15 \\
\end{array}$ & $\begin{array}{l}4.09 \\
4.76 \\
\end{array}$ & $\begin{array}{l}.001 \\
.05 \\
\end{array}$ \\
\hline
\end{tabular}


by number/form. For purposes of exposition, we will use the labels XL (extra large), L (large), M (medium), and $S$ (small) to refer to the four levels of relative size (see Figure 2).

\section{Accuracy}

The significant effect of triplet upon accuracy was due to higher error rates with the smaller patterns. Since the overall analysis of errors did not reveal a significant interaction between triplet and type of comparison stimulus, we can conclude that whether number or spacing remains the same makes little difference for accuracy in the categorization task. Therefore, in the remaining analyses, accuracy will be largely ignored.

\section{Similarity Judgments}

To determine the effects of type of comparison stimulus, triplet, and their interaction as a function of overall stimulus size, ANOVAs were performed on the data from each "size" panel in Figure 3A. For the XL stimulus, the effect of type of comparison stimulus was marginally significant $[F(1,15)=4.46, p<.1]$ and the interaction between type of comparison stimulus and triplet was significant $[F(3,45)=17.12, p<.001]$. For the L stimuli, the effect of type of comparison stimulus was significant $[F(1,15)=8.6, p<.025]$, and the interaction between type of comparison stimulus and triplet was significant $[F(2,30)=14.84, p<.001]$. For the $M$ stimuli, the main effect of type of comparison stimulus was significant
$[F(1,15)=4.99, p<.05]$, but the interaction was not $[F(1,15)=2.61, p>.1]$. There was no effect of type of comparison stimulus with the smallest patterns.

Statistical information that could be compared with Goldmeier's frequency data (i.e., what proportion of subjects favor same-number or same-spacing stimuli?) was generated by testing for effects of type of comparison stimulus for each of the 10 triplets. The results of these ANOVAs ${ }^{4}$ are shown in Figure $3 \mathrm{~A}$ as probability values. This analysis, together with the preceding ANOVAs reveals that the crossover point (the point at which material emerges as perceptually salient, and, therefore, proportional enlargement is no longer dominant) varies with the overall size of the stimuli or, in other words, is a joint function of number and spacing.

With our XL (crossover = 5/9) and L (crossover = 3/55\%) stimuli, the crossover point is in the range suggested by Goldmeier's observations, between $3 / 5$ and $7 / 15$. However, with our $M$ (crossover $=3 / 5$ ) and $S$ (crossover $=2 / 3$ ) stimuli, the crossover point occurs at smaller numbers. It should be kept in mind that, as number stays constant, moving from $X L$ to $S$ involves changes in spacing, and it is therefore legitimate to question whether the shifting crossover point as a function of size is best characterized in terms of size or spacing.

\section{Categorization}

ANOVAs were performed on the RTs from each size panel in Figure 3B. For the XL and L panels, there were
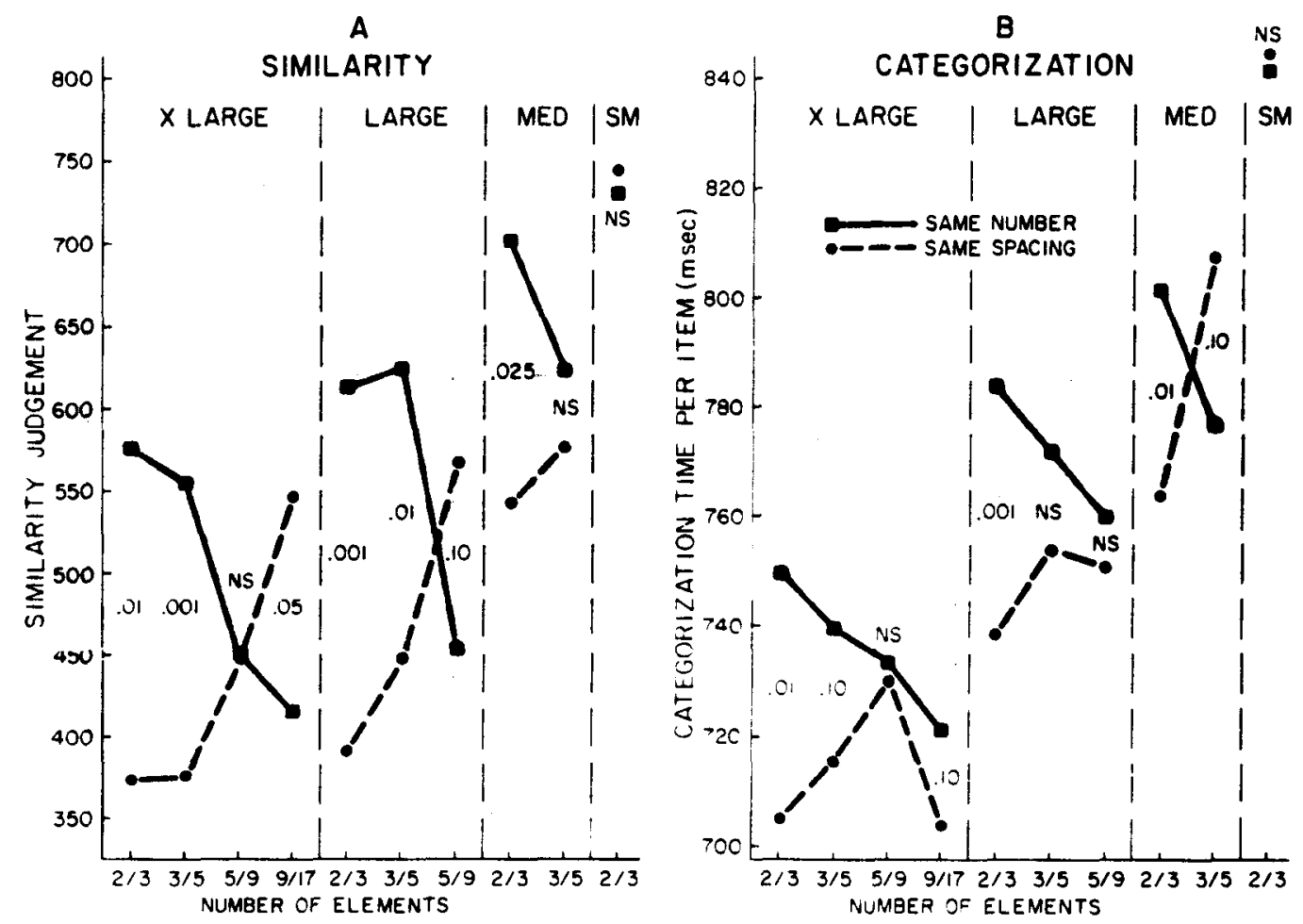

Figure 3. Similarity (a) and categorization time (b) as a function of stimulus size (panels correspond to dingonals in Figare 2) and type of comparison stimulus. 
main effects of type of comparison stimulus $[F(1,15)=$ $22.21, \mathrm{p}<.001$, for $\mathrm{XL} ; \mathrm{F}(1,15)=6.87, \mathrm{p}<.025$, for $\mathrm{L}]$, but the interactions between type of comparison stimulus and triplet were not significant $[F(3,45)=2.07$, p > .1, for XL; $F(2,30)=1.5$, for $L]$. For the M panel, the interaction between type of comparison stimulus and triplet was significant $[\mathrm{F}(1,15)=12.84, \mathrm{p}<.01]$, whereas there were no differences with the smallest size. Visual comparison of the pattern of results with RT and similarity reveals a generally consistent pattern, with the exception of the most numerous stimuli in the $\mathrm{XL}$ and $\mathrm{L}$ panels, where a crossover fails to emerge in RT as number increases.

The results of the analysis of the RTs for each combination are shown as probability values. If these data are interpreted in terms of the point at which same number ceases to be slower than same spacing, the pattern of change with size (panel) is quite similar to that observed in similarity $(5 / 9$ for XL; $3 / 5-5 / 9$ for $L ; 2 / 3-3 / 5$ for $M ; 2 / 3$ for $S)$. The major discrepancy between similarity and RT appears with the XL $\% / 17$ and the $\mathrm{L} \%$. Since these failures of RT to demonstrate crossover are interesting and theoretically important, it is prudent to examine accuracy in the corresponding conditions to determine if the anomalously rapid RTs in the same spacing pairs were produced by speed-accuracy tradeoffs. For the XL $9 / 17$ stimuli, accuracy on same spacing and same number pairs was the same (.019 and .019 proportion errors, respectively); for the L $5 \%$ stimuli, accuracy with same spacing was nonsignificantly better (.010) than on same number pairs (.021), so in neither case is the lack of crossover in RT due to a speed-accuracy tradeoff.

\section{DISCUSSION}

There are two significant empirical contributions we would like to emphasize. The first contribution derives from our parametric extension of Goldmeier's observations. With the large and extra large stimuli, our similarity data are in very close agreement with those of Goldmeier, in spite of differences in stimulus construction (lines vs. dots) and procedure. The consistency between the two methods suggests a powerful phenomenological effect. Nevertheless, our parametric manipulation shows something that is only hinted at in Goldmeier's paper. Subjects' similarity judgments show that the emergence of material as a perceptually salient dimension is a joint function of the number and spacing of the elements in a linear array. A modification of Goldmeier's Rule 2 seems warranted: Material begins to emerge at smaller numbers when the spacing between elements or overall size is very small. $^{5}$

The second empirical contribution of our study lies in the comparison of the performance and phenomenological measures. By and large, these are in good agreement. However, the failure of RT to show the crossover effect with the most numerous, most closely spaced stimuli is a salient exception. Although we cannot provide a defini- tive explanation for the observed RT/similarity discrepancy, consideration of the degree to which a form and the elements that compose it are perceptually integral or separable (Garner, 1974) suggests one. In the following discussion, we draw upon the ideas of Garner (1974), Goldmeier (1936/1972), Julesz (1975), Kimchi and Palmer (1982), and Marr (1982). In particular, we are grateful to Kimchi and Palmer for stressing the two functional roles that elements play in the form/material distinction. However, following Marr (1982), we will view these roles within their natural ecological context.

Objects in the world have a global form that is composed of its various parts in relation. Objects are also made out of various " $s$ tuff" or material. Thus, for example, a human body has a global cylindrical form, composed of a nested hierarchy of other cylinders-trunk, limbs, neck, and head (Marr, 1982). But a body is also made out of various chemical materials. Now there is a clear distinction between the "parts" of a body and the "material" out of which it is made. In the natural world, the object's parts rarely coincide with the elements out of which it is composed. However, in our "minimal" line stimuli, the individual dots can, and do, serve both as parts of the global "line" form and as the elemental material of which the line is composed. As a result, there are two competing organizations for the functional role of the dots in the figure, and both the perceptual similarity and the sorting task demonstrate-though differently-the shift in the relative salience of dots-as-parts and dots-as-material at, what we have called, the crossover point.

In order to better understand the nature of this shift, it is useful to keep distinct two properties of our line stimuli. The first is the line itself. Depending on the number of dots and the spacing of the dots, the global appearance of the line can be more or less salient, or "real." Generally speaking, when the line is long and composed of few dots, it appears more "imaginary" than real. Its reality increases as the density increases, or the spacing decreases. ${ }^{6}$ The second property of our stimuli is the salience of dots as "individuals." When the line is composed of few dots that are distantly spaced, the dots are seen as individuals whose organization composes the line (cf. Kimchi \& Palmer, 1982, Experiment 5). But when the dots are closely packed, or increased greatly in number, they lose their status as individual parts of the figure, and become the material of which the line is made. Hence, the status of dots as individuals changes from "real" to "imaginary" as their role in the figure shifts from parts of a line to material out of which it is composed. This is the crossover point.

We can now discuss the differential effect of "crossover" on similarity and performance. Prior to crossover, the line stimuli form a nested hierarchy of parts whose global form is the line and whose elemental parts are the individual dots. The fewer the number of dots and the further the spacing of dots, the more salient are the dots and the less salient the line of these "integral" (Garner, 1974) stimuli. To the extent that the individual dots dominate 
the overall appearance of the stimuli, similarity is particularly sensitive to variations in dot number, and greater similarity is awarded to the comparison stimulus that maintains the same number of individuals. As the number of individuals increases, or the spacing decreases, this relative preference decreases as we approach crossover. This same parameter has a comparable effect on the sorting task. Because, prior to crossover, the individual dots are integral parts of the line stimuli, sorting decisions are affected by their number. The more salient the number of dots are in the stimuli, the more this "redundant" cue can be used-when different-to decrease sorting time, and the more it will interfere-when the same-with judgments based on line length alone. However, beyond crossover, these two tasks dissociate. Once the functional role of individual dots in the pattern shifts from being parts in an integral organization to being mere material, they become "separable" (Garner, 1974) from the figure itself (Goldmeier refers to "phenomenal separation"), and this leads to a differential outcome on the two tasks. On the similarity task, subjects make the "cognitive" judgment that the lines composed of the same material are more similar to each other than stimuli composed of different material. This may occur because material is often an important cue to an object's identity (see Marr, 1982, chap. 3). However, in the sorting task, subjects are now able to make their decisions purely on line length without the interference or assistance of the independently coded "material" dimension. Hence, we would expect no difference in sorting time as a function of number of elemental dots beyond crossover. Indeed, as Kimchi and Palmer (1982, pp. 534-535) point out, the global form may then be perceived prior to its parts, and this could lead to faster RTs beyond crossover. Our results are consistent with this principle.

This same sort of explanation can be applied to some of the results of the Kimchi and Palmer (1982) study. Although their first four experiments provide the necessary converging evidence for their, and our similar, interpretation of the fifth experiment, it is only on this experiment that the present discussion will focus. In this experiment, subjects were asked to select among four verbal descriptions of a pattern that stressed the global form, the global form plus the number of elements, the elements, or the elements and their number. Kimchi and Palmer found that subjects preferred verbal descriptions that emphasized properties of the pattern that the previous experiments had found to be perceptually salient. As in our experiment, the elements in their study served both as "parts" of the global form and as the "stuff" of which it was made. These two functional roles competed in their relative salience to the subject. When the number of elements were few, they were perceived as individuals arranged into a global organization. Hence, subjects preferred descriptions that stressed the elements as well as their numbers. However, as in our crossover effect, when the number of elements was large, their role as material dominated. They were no longer perceived primarily as individuals whose numbers were important, but as stuff out of which the global figure was made. Hence, subjects preferred descriptions that stressed the global form and ignored the number of elements. That two functional roles are involved in these results is suggested by another aspect of their data. They found that the "consistency" of the local and global forms (e.g., a triangle composed of triangles vs. squares) affected choice of description only when the number of elements was small. Under these circumstances, inconsistency led to an increased salience of elements. No comparable effect of consistency occurred for the many-element figures. This is again suggestive evidence that elements are "integral" parts of the figure when their numbers are few, but are "separable" material when their numbers are many (Kimchi \& Palmer, 1982, p. 534). Our explanation of the failure of RT to show a crossover effect in our study converges with this view. Firm conclusions, however, require a more direct experimental attack.

\section{REFERENCES}

GARNER, W. (1974). The processing of information and structure. Hillsdale, NJ: Erlbaum.

GoldmeIER, E. (1972). Similarity in visually perceived forms. Psychological Issues, 8(Whole No. 29). (Original work published 1936)

JULESZ, B. (1975). Experiments in the visual perception of texture. Scientific American, 232, 34-43.

KIMCHI, R., \& PALmer, S. E. (1982). Form and texture in hierarchically constructed patterns. Journal of Experimental Psychology: Human Perception and Performance, 8, 521-534.

MARR, D. (1982). Vision. San Francisco: Freeman.

PALMER, S. (1977). Hierarchical structure in perceptual representation. Cognitive Psychology, 9, 441-474.

Posner, M. I. (1978). Chronometric explorations of mind. Hillsdale, NJ: Erlbaum.

WOODWORTH, R. S., \& SCHLOSBERG, H. (1954). Experimental psychology: Revised edition. New York: Holt, Rinehart \& Winston.

\section{NOTES}

1. It should be noted that our stimuli, and those used by Goldmeier in his Figures 24 and 25, are not strictly proportional enlargements, since the dots do not change size as spacing does.

2. Nine different possible locations were selected from a centered, $3 \times 3$ grid with a horizontal spacing of approximately $5^{\circ}$ and a vertical spacing of about $4^{\circ}$. If stimulus lines were always displayed at the same screen coordinates, stimulus categorization could be accomplished merely by observing if there were or were not dots in a particular screen region (i.e., beyond the boundaries of the smaller stimulus). Variation in location was used to thwart this strategy.

3. Every stimulus pair that was presented to our subjects for categorization or similarity judgments consisted of two lines of dots that differed in overall extent by a 2:1 ratio. Moreover, each subject in the experiment experienced stimulus sizes over an 8-to-1 range. If Weber's law applied over this considerable range of stimulus sizes, then one would not expect stimulus size (recall that triplets on each diagonal in Figure 2 are the same in overall extent) to produce very large effects. Inspection of our data suggests otherwise. Since size differences across the 10 triplets were produced by changing spacing, number, or both parameters, it is not possible to look at the effects of size without some variation in these factors. Examination of the results shown in Figure 3 reveals that the increases in both similarity and RT with decreasing overall size is 
about the same when all stimuli of a given size are examined (compare panel averages) or when size changes are produced by changes in spacing (leftmost data in each panel) or number (rightmost data). Clearly, Weber's law does not hold. Interestingly, each halving of size adds a relatively constant amount to categorization time and similarity judgments.

4. Three-way ANOVAs were performed with base spacing a betweensubjects factor, and block and type of comparison stimulus within-subject factors. The $p$ values are based on the $F$ ratios for the main effect of type of comparison stimulus.

5. Goldmeier foreshadowed this conclusion: "It must be admitted that the effect of number cannot be completely isolated from the effect of linear extent ... it is thus conceivable that the relative diminution in the dimensions of elements . . . by itself causes the separation into material and form" (p. 50). Kimchi and Palmer (1982) also provide a similar elaboration of Goldmeier's rules.

6. It is of some interest that, in a pilot study for their Experiment 5, half of Kimchi and Palmer's (1982, p. 533) subjects referred to their comparable stimuli as having an "imaginary" form.

(Manuscript received October 1, 1984; revision accepted for publication April 1, 1985.) 